\title{
Wie man auf neue Gedanken
} kommt

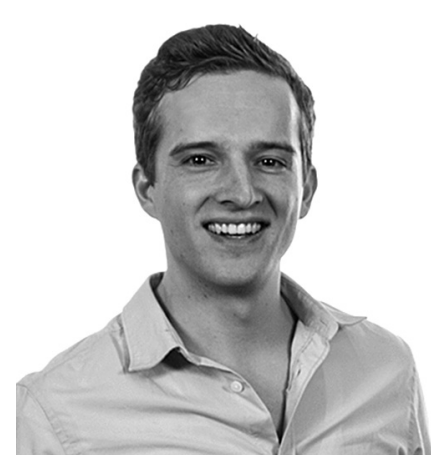

VON JOHANNES MEYER Johannes Meyer ist Design Thinking Coach, Moderator und Innovationsberater. Nach dem Studium war er interner Innovation Consultant bei SAP und arbeitete an Software-Innovationen im Bereich Banken, Rechnungswesen und Einzelhandel. Derzeit gestaltet er als Program Manager der HPI Academy GmbH Workshops und Beratungen, die Design Thinking nutzen, um Unternehmen auf dem Weg zu neuen Produkten, Prozessen und einer innovativeren Arbeitskultur zu unterstützen. Neben seiner Arbeit ist er Sänger einer Rockband in Berlin. www.hpi-academy.de

\author{
Design Thinking ist eine Möglichkeit zur Entwicklung \\ neuer Ideen in allen Lebensbereichen. Die Methode \\ basiert auf der Überzeugung, dass wahre Innovation nur \\ dann geschehen kann, wenn starke multidisziplinäre \\ Gruppen sich zusammenschließen, eine gemeinschaftliche \\ Kultur bilden und die Schnittstellen der unterschiedlichen \\ Meinungen und Perspektiven erforschen
}

Der Ruf nach mehr »Innovation « zieht sich durch viele Branchen. Schnelle Produktzyklen, sich beständig verändernde Rahmenbedingungen und der Wunsch aus "weniger mehr zu machen « steigern den Druck auf Unternehmer und Führungskräfte.

Aber wie lässt sich die Suche nach innovativen Ideen, Prozessen oder Erlebnissen strukturiert angehen? Eine neue, dem Design angelehnte Methode aus dem Umfeld des Silicon Valley setzt sich derzeit verstärkt in deutschen Organisationen durch: Design Thinking.

Sehen Sie sich um: Alles was nicht die Natur gestaltet hat, ist »designt«. Dazu gehören nicht nur sichtbare und anfassbare Designs, sondern auch Erlebnisse oder Prozesse: Der Geruch bei H\&M, die Begrüßung bei Starbucks, die Art wie Sie ein Flugticket kaufen oder der Prozess ihrer Steuererklärung - jemand hat entschieden, dass diese Dinge sich genau so anfühlen und funktionieren, wie sie es tun.

Das ist das breite Verständnis des Designbegriffs, das auch dem Begriff »Design Thinking « zugrunde liegt. Wenn Sie in Ihrer Arbeit an der Gestaltung von Erlebnissen beteiligt sind - Erlebnisse ihrer Kunden oder Nutzer, Ihrer Mitarbeiter oder Geschäftspartner -, dann kann Design Thinking spannende Impulse für Wege zu Problemlösungen, aber vor allem für die proaktive Gestaltung und Verbesserung solcher Erlebnisse bieten.

Design Thinking ist eine Problemlösungs-Kultur, die durch den bewussten
Einsatz von Teams, Räumlichkeiten und einem iterativen Arbeitsprozess geprägt ist. Erwähnungen des Begriffs gibt es bereits seit den 1980er Jahren, in den 1990ern wurde das methodische Gerüst für Innovation im Umfeld der Universität Stanford beschrieben und entwickelt.

Unternehmen wie IDEO begannen, Innovation branchenübergreifend als Dienstleistung anzubieten und durch Ihre besondere Herangehensweise an Problemstellungen aufzufallen. Neben Projekten wie dem ersten faltbaren Computer, der ersten Apple-Mouse, der Ausgestaltung von Kinderkrankenhäusern oder Wohnkonzepten für Kriegsversehrte waren die Unternehmensgründer auch in der Lehre zu engagiert und brachten die erste "School of Design Thinking « auf den Weg.

Diese »D-School «, später von SAPMitgründer Hasso Plattner großzügig unterstützt, hat inzwischen ein Schwesterinstitut in Potsdam, an dem Studenten und erwachsene Lerner anhand echter, relevanter Fragestellungen den Umgang mit der kollaborativen Innovationskultur trainieren. Wie die drei Kernelemente Räume, Teams und Arbeitsprozesse sich dabei manifestieren, soll im Folgenden skizziert werden.

\section{Erstes Kernelement: Räumlichkeiten}

Das Umfeld, in dem wir arbeiten, beeinflusst unser Verhalten und unseren Umgang miteinander. Dieser einfache Um- 
stand kann in der Innovationsarbeit genutzt werden. Räumliche Flexibilität unterstützt auch Flexibilität der Gedanken, weswegen an beiden D-Schools Möbel rollbar, verschiebbar und verschieden kombinierbar sind. Teamarbeitsplätze sind in Stehhöhe und hierarchiefrei angelegt - keine schweren Konferenztische mit Stirnseiten für Chefs, sondern kleine Arbeitsnischen, die das Zusammenarbeiten in kleinen Gruppen unterstützen.

\section{Zweites Kernelement: Teams}

Innovationen und Antworten auf komplexe Fragestellungen erfordern meist eine Vielzahl an Perspektiven, Expertisen und Arbeitsweisen. Deshalb setzt Design Thinking nicht auf den einzelnen Erfinder, sondern auf die Innovationskraft multidisziplinärer Gruppen von idealerweise vier bis sechs Personen. Wenn anerkannt wird, dass jeder in seinem Feld Experte ist, entsteht ein natürlicherweise hierarchiearmes, kollaboratives Umfeld, das das Potential jedes einzelnen nutzt. In der Praxis ist die Interaktion in solchen Teams ein komplizierter Prozess und oftmals das Schwierigste an einer Design-ThinkingArbeitskultur.

\section{Drittes Kernelement: Arbeitsprozesse}

Design Thinking orientiert sich an einem breiten Verständnis des Designbegriffs und dem Denkprozess, dem Designer oftmals intuitiv folgen. Dabei gilt es vor allem, das Lernen über ein zu lösendes Problem von der Lösungsentwicklung zu trennen und Einschränkungen nicht immer gleichzeitig zu betrachten, sondern sequentiell zu bearbeiten. Für die praktische Arbeit und die Orientierung im Team wird dieser Prozess in verschiedenen Phasen oder Modi beschrieben. Dabei gibt es verschiedene Modelle; das von der Potsdamer D-School interpretierte und damit in Europa richtungsweisende nimmt eine Einteilung in sechs Phasen vor:

Verstehen: die Orientierung des
Teams untereinander, aber auch
in der Fragestellung. Da Projekte in der
Realität oft mit impliziten Lösungen
starten, wird hier darauf geachtet, dass
Wissen von Hypothesen getrennt wird
und dass das Team sein zu bearbeitendes
Territorium und die betroffene Nutzergruppe absteckt. Oftmals wird das zu bearbeitende Problem dann in einem Satz beschrieben, zum Beispiel: "Wie können wir die Notaufnahmen von Krankenhäusern besser für Menschen mit Demenz gestalten? «(Dies ist eins der Projekte, die in der Vergangenheit in Potsdam bearbeitet wurden.)

2. Beobachten: In dieser Phase bedienen sich die Teams ethnographischer Forschungsmethoden und bauen Empathie für die beteiligten Zielgruppen auf. Dabei wird weniger auf Fragebögen und aufwändige Studien gesetzt als auf Beobachtungen und intensive Interviews. Im genannten Beispiel zu Notaufnahmen bei dementen Patienten führte das Team Gespräche mit Betroffenen, Angehörigen und medizinischem Personal, beobachtete Arbeitsabläufe und begab sich selbst in die Situation des Nutzers und ließ sich in der Notaufnahme aufnehmen. Ziel dieser Phase ist eine tiefe Empathie für die Motivationen, Gefühle und Entscheidungswege von Nutzern.

3. Sichtweise definieren: In Beobachtungsphasen ergeben sich meist eine ganze Reihe von Innovationsräumen, in denen Dinge verbessert oder komplett neu gedacht werden könnten. Die Synthese dieser meist unstrukturierten Möglichkeiten hin zu einer neuen, fokussierten Herausforderung für das Team ist ein gedanklicher Zwischenschritt der hinter den meisten Designs steht. So wie ein Autohersteller aus Kenntnis seiner Zielgruppe entscheiden mag, dass die nächste Version des Wagens »mehr Aggression « ausstrahlen soll, so könnte der Fokus im beschriebenen Projekt folgender sein: »Wir wollen verhindern, dass demente Patienten aus Nervosität weglaufen." Oftmals wird die Sichtweise oder der »Standpunkt « durch kompakte Informationsträger unterstützt: Sogenannte Personas, also sehr genaue prototypische Beschreibungen des Zielnutzers mitsamt Namen, Alter, Zielen und Wünschen helfen besonders solchen Teams, die Lösungen für Zielgruppen schaffen sollen, denen sie nicht selber angehören. Ideen generieren: Die Phase der
mit der viele Innovationsmethoden oftmals gleichgesetzt werden, ist im Design Thinking ein sehr kurzes Intervall. Die Kombination unterschiedlicher Sichtweisen in einem Team kann zu Lösungen führen, die über die Ideen jedes Einzelnen hinausgehen. Damit diese gegenseitige Inspiration auch wirklich funktioniert, setzen viele Teams Assoziationstechniken ein, die darauf abzielen zunächst eine große Vielfalt an Ideen zu generieren und das Bewerten, Priorisieren und Auswählen danach vorzunehmen. Im erwähnten Projekt kristallisierte sich aus einer Vielfalt an Ideen das Konzept einer „Haube“ heraus, die sich an Krankenbetten über dem Kopfteil montieren lässt und auf Patienten beruhigend wirken soll.

\section{Was versteht man eigentlich unter "Design Thinking»?}

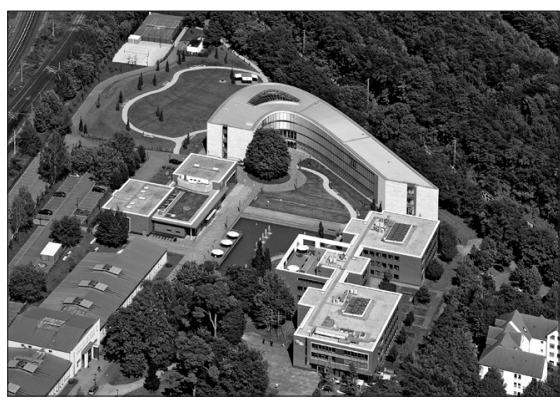

Design Thinking wird eine Vorgehensweise genannt, die zur Lösung von Problemen und zur Entwicklung neuer Ideen führen soll. Die Methode basiert auf der Annahme, dass Probleme besser gelöst werden können, wenn Menschen unterschiedlicher Disziplinen zusammenarbeiten, gemein- sam eine Fragestellung entwickeln, die Bedürfnisse und Motivationen von Menschen berücksichtigen, und dann Konzepte entwickeln, die mehrfach geprüft werden. Das Verfahren orientiert sich an der Arbeit von Designern, die als eine Kombination aus Verstehen, Beobachtung, Ideenfindung, Verfeinerung, Ausführung und Lernen verstanden wird. Gefördert werden Erforschung und Umsetzung dieses Konzepts durch den Milliardär Hasso Plattner. Prinzipien des Design Thinking werden seit 2005 am Hasso Plattner Institute of Design, der sogenannten »d.school«, gelehrt (vgl. Foto). Im Oktober 2007 nahm die School of Design Thinking am Hasso-PlattnerInstitut in Potsdam den Studienbetrieb auf.

Quelle: www.wikipedia.org 
5. Prototypen entwickeln: Eines der Kernprinzipien im Design Thinking ist "Zeigen, nicht erzählen«. Innovative Produkte oder Abläufe enthalten immer eine Reihe von Unsicherheiten. Diese gilt es prototypisch in ein abfassbares Format zu bringen, um mehr über sie zu lernen. Als »Prototyp « gilt dabei alles, was auf eine günstige Art einzelne Hypothesen hinter einer Idee konkretisiert und sich mit Nutzern oder Experten in Kontakt bringen lässt. Das können Vorstufen eines Produkts sein, aber auch Prototypen von einzelnen Interaktionen, Geschäftsmodellen oder Dienstleistungen.

6.

Testen: Prototypen können eine Reihe von Zielen verfolgen; sie können Gespräche in Teams vermitteln oder bei der Darstellung einer Idee helfen. Zumeist sind sie jedoch für eine spezifische Art von Test-Szenario gedacht, in dem das Team mehr über seine Idee lernen möchte. sind und das gerade im umkämpften Personalmarkt der Branche ein wichtiges Thema ist. Ist es ein perfektes Erlebnis? Bestimmt nicht. Wie würden Sie also verfahren, wenn Sie hier Innovation schaffen wollen? Denken Sie zunächst darüber nach, wie Sie für einen kurzen Projektzeitraum ein Team zusammenstellen können, das viele unterschiedliche Perspektiven vereint. Nicht nur Personalexperten, sondern vielleicht bewusst auch Fachfremde, zum Beispiel Azubis. Stellen Sie diesem Team eine offene Frage: "Wie können wir das Einstellungs-Erlebnis für neue Mitarbeiter verbessern?«

Um Empathie aufzubauen wird das Team dann anfangen zu forschen: Neue Mitarbeiter nach Ihrer Sichtweise fragen, ergründen, welche Fragen und Bedenken an welchen Punkten des komplexen Erlebnisses auftauchen und Punkte identifizieren, an denen vielleicht sogar mit einfachen Mitteln Verbesserung geschaffen werden kann. Vielleicht geht es

\section{"Design Thinking setzt nicht auf den einzelnen Gestalter, sondern auf die Innovationskraft multidisziplinärer}

\section{Gruppen«}

Ideen können scheitern - sollten dies jedoch möglichst früh und günstig tun. Der Design-Thinking-Prozess unterstützt eine Kultur des Scheiterns und ist deshalb kein geradliniger Arbeitsablauf, sondern ein Zyklus an Iterationen, die durch beständiges Lernen und Ausprobieren zu innovativen Ergebnissen kommen.

\section{Einsatz in der Sozialwirtschaft - ein Beispiel}

Lassen Sie uns nach diesem Umreißen der Werte hinter Design Thinking ein paar Anknüpfungspunkte für die Sozialwirtschaft vorschlagen. Natürlich sind Sie selbst hier die Experten und kennen sich mit Ihren Rahmenbedingungen und Fragestellungen am besten aus.

Alle Fragestellungen, die sich mit Menschen und menschlichen Erlebnissen beschäftigen, passen zu Design Thinking. Nehmen wir die Einstellung neuer Mitarbeiter - ein komplexes Erlebnis, an dem viele Gestalter beteiligt nur um eine zusätzliche Nachricht, eine Information oder ein Feedback.

In der Prototypen-Phase wird das Team dann solche Maßnahmen ausprobieren oder improvisieren (damit beispielsweise eine neue Webseite zunächst nicht aufwändig produziert werden muss), und auf die Reaktion der Nutzer testen. Wenn das Team genügend Freiräume für seine Recherche und seine Experimente bekommt, können so in kurzen, abgesteckten Zeiträumen spannende Vorschläge entstehen, die das beschriebene Erlebnis positiv beeinflussen. Natürlich lässt sich die gleiche Herangehensweise auch auf andere Fragestellungen übertragen: Sei es das Finden neuer Mitarbeiter, einzigartige Angebote Ihrer Einrichtung für Kunden oder Betroffene, Kommunikationsprozesse oder Feedback-Modelle - wenn Sie die bestehenden »Designs « nicht als gegeben hinnehmen und einen frischen Blick darauf wagen, werden Sie Dinge entdecken, die Sie derzeit nicht ahnen.

\section{Resümee}

Design Thinking ist mittlerweile im deutschsprachigen Raum in einer Reihe von Unternehmen und Institutionen angekommen und es gibt spannende Beobachtungen zu den Reaktionen von Management und Mitarbeitern abseits der entstehenden Innovationen. So wird die Einbindung von Mitarbeitern als Team-Mitglieder, aber auch als befragte Nutzer als Wertschätzung und Beteiligung an Lösungsfindungen sehr positiv aufgenommen (»Ich werde hier gefragt«). Außerdem stärkt Empathie über Hierarchieebenen hinweg den $\mathrm{Zu}$ sammenhalt und die Glaubwürdigkeit von Führungskräften (»Der interessiert sich wirklich für mich «). Schlussendlich ist es für viele Teams befreiend, weg vom »Das geht sowieso nicht « endlich mal an das zu denken, was vielleicht möglich gemacht werden könnte und in dieser Haltung von Ihren Führungskräften unterstützt zu werden.

Jede Institution hat eine gewachsene und sicherlich auch erfolgreiche Kultur und das Ziel soll nicht sein, sich all das von einer Innovationsmethode umkrempeln zu lassen. Aber vielleicht kann Ihnen Design Thinking Anregung geben, Dinge zu tun, die den Alltag aufbrechen und helfen, den Blick auf Ihr Umfeld frisch zu halten.

Ein pragmatischer Vorschlag: Stehen Sie jetzt auf, sprechen Sie die erste Kollegin oder den ersten Mitarbeiter an, den Sie treffen, und fragen Sie mal, wie Ihr Haus eigentlich wahrgenommen wird. Was genau sie oder er an seiner Arbeit schätzt und was eher schwierig ist. Lassen Sie sich von einem hervorragenden und von einem grauenhaften Arbeitstag aus der Vergangenheit erzählen und fragen Sie nach dem Warum. Machen Sie Notizen und nutzen Sie die Informationen als Start Ihres Empathie-Projekts.

\section{Literaturtipps}

Johannes Meyer \& Jochen Gürtler. 30 Minuten Design Thinking. Gabal, 2013.

Jürgen Erbeldinger \& Thomas Ramge. Durch die Decke denken: Design Thinking in der Praxis. Redline, 2013.

Tim Brown. Change by Design. How Design Thinking Transforms Organizations and Inspires Innovation. Harper Business, 2009 